\title{
Microdialysis-directed Intra-tumor Pharmacokinetic Modeling of Methotrexate in Mice and Humans
}

\author{
Helal H. Alsulimani ${ }^{1}$, Jonghan Kim ${ }^{1}$, Shabnam N. Sani ${ }^{2}$ \\ ${ }^{1}$ Northeastern University, Department of Pharmaceutical Sciences, Boston, MA, USA. ${ }^{2}$ Western New England University, \\ College of Pharmacy, Department of Pharmaceutical \& Administrative Sciences, Springfield, MA, USA.
}

Received, May 27, 2016; Accepted, June 21, 2016; Published, June 30, 2016.

\begin{abstract}
Purpose. To develop a quantitative pharmacokinetic model to characterize the disposition of methotrexate (MTX) at tumor site in tumor-bearing mice and to predict MTX concentrations in the human tumor. Methods. The plasma profiles of MTX were obtained from normal mice, while microdialysis technique was employed to characterize the time course of MTX in tumor from breast tumor-bearing mice. Disposition profiles of plasma and tumor were analyzed by a hybrid physiologically-based pharmacokinetic (hPBPK) model that incorporates physiologically-relevant parameters such as tumor blood flow and volume, while plasma concentrations were used as a forcing input into the vascular-interstitial spaces of the tumor. The plasma profiles were initially described by a biexponential decay model to obtain a forcing function that enters into the vascularinterstitial spaces in the tumor. Using a defined forcing function, the tumor free concentrations were fitted to the hPBPK model. Based on the model developed, sensitivity analysis was conducted with a perturbation of PK parameters to predict different scenarios of intratumoral MTX transport. The relevant physiological PK parameters from the mouse model were then scaled-up and utilized to simulate human tumor concentrations. Results. The mouse hPBPK model adequately characterized the concentration-time profiles of MTX in both plasma and tumor and produced various transfer rate constants between plasma and tumor. Our model was also able to reasonably predict MTX concentrations in the human tumor when human physiological data were utilized. Conclusions. The hPBPK model was able to quantitatively characterize the atypical transport of MTX in the tumor, supporting the idea that microdialysis is a valuable tool to study tumor biodistribution of drugs and to predict tumor concentrations in humans based on the pre-clinical data. This information can ultimately aid in the development of anticancer drugs with improved PK profiles.
\end{abstract}

This article is open to POST-PUBLICATION REVIEW. Registered readers (see "For Readers") may comment by clicking on ABSTRACT on the issue's contents page.

\section{INTRODUCTION}

The therapeutic efficacy of anticancer drugs is critically dependent upon the maintenance of adequate drug concentrations in the "target" site. Because it is difficult to determine the drug concentration at the site of action, the plasma concentration is generally used as a reference index for therapeutic purposes $(1,2)$. However, there is a large disparity in drug concentrations between plasma and tumor due to inadequate drug transport and delivery to tumors and resistance mechanisms developed by tumor cells $(3,4)$. Suboptimal target site concentrations can have important clinical implications, as this can be a potential explanation for some therapeutic failures (5-7). Thus, the development of anticancer drugs to intended target sites remains a challenging task that requires tremendous amount of financial and scientific investment (8).

In the past 30 years, despite many advances in molecular and targeted anticancer therapeutics, most anticancer drug candidates have continued to fail in clinical trials and the success rate of drug development in oncology remains significantly lower than other therapeutic areas (9). Chemotherapeutical failure in cancer patients often occurs due to various reasons. Of the many challenges, one of the likely

Corresponding Author: Dr. Shabnam Sani, Western New England University, College of Pharmacy, Department of Pharmaceutical \& Administrative Sciences, 1215 Wilbraham Road, Springfield, MA 01119, USA; ssani@,wne.edu 
reasons for the high attrition rate is the failure in pharmacokinetics and pharmacodynamics, such as inadequate tumor drug concentrations leading to insufficient drug efficacy (10). It is known that adequate delivery of chemotherapeutic agents to the target site is a prerequisite for antitumor activity (11, 12). Unfortunately, concentration measurements in the target site have not been a routine practice during the standard drug development process and certainly has not been given enough consideration because regulatory requirements have not mandated target site characterization. Optimal dose and dosing regimen selection in oncology still remains semiempirical, making the existing oncology schedules far from being optimized (13). Hence, the characterization of concentration-time profiles in the target site (tumor) in addition to the plasma will definitely improve our understanding of plasmatumor transport of anticancer drugs.

Methotrexate (MTX) has played a crucial role in cancer chemotherapy in combination with other chemotherapeutic agents in the treatment of various solid tumors such as breast cancer, acute lymphocytic leukemia, osteogenic sarcoma, choriocarcinoma, lung cancer, and chronic myeloid leukemia (14). Some classify MTX among the most effective drugs for treatment of non-malignant disease and is often considered the "gold standard of therapy". Although MTX is an old drug approved by the FDA in 1953, many studies have reported high inter-subject variability in clinical response to MTX due to the inconsistent relationship between plasma concentrations and intra-tumor levels of the drug $(15-17)$ and thus, there has been a growing interest in recent decades to apply microdialysis methodolog to evaluate the disposition of anticancer agents at the target site such as the tumor xenografts, CNS, and in patients with accessible tumors and to develop more informative quantitative PK models $(15,18,19)$. High inter-subject variability in clinical response to MTX, the inconsistent relationship between plasma concentrations and intra-tumor levels of the drug, and the scarcity of the information about its accumulation in tumors and normal tissues provided us with the impetus to develop a more comprehensive pre-clinical PK model of MTX in breast tumor that can subsequently be scaled to predict human breast MTX concentrations. There have been limited number of reports that have characterized plasma and tumor exposure of MTX using microdialysis in clinical and preclinical settings $(15,16,18,19)$. These efforts have included non-compartmental and system-based modeling approaches to study MTX distribution. Most of the other previous attempts have relied solely on MTX plasma concentrations to build up compartmental models $(20,21)$. These models have described MTX disposition as a two- or three-compartment model since the log-scale plasma concentration profiles showed more than one declining phase after an IV bolus injection of MTX. However, no investigation has attempted to develop a comprehensive modeling approach to quantitatively characterize atypical transport of MTX in the tumor. Therefore, the objective of this study is to develop a more comprehensive quantitative pharmacokinetic model to characterize the disposition of MTX at the tumor site using microdialysis in a tumor-bearing mouse model and to predict tumor concentrations in humans based on the pre-clinical data.

\section{MATERIALS AND METHODS}

\section{Microdialysis System}

Linear microdialysis probes (CMA30, 10mm cuprophane membranes, $6000 \mathrm{Da}$ cutoff) and peripheral perfusion fluid $\mathrm{T} 1$ were purchased from CMA Microdialysis (Chelmsford, MA). The peripheral perfusion solution (an isotonic, sterile solution composed of $147 \mathrm{mM} \mathrm{NaCl}, 4 \mathrm{mM} \mathrm{KCl}$, and $2.3 \mathrm{mM} \mathrm{CaCl} 2$ ) was delivered, using a $1 \mathrm{~mL}$ glass microsyringe at ambient temperature, by a Harvard apparatus pump, and the perfusates were collected manually at specified time intervals.

\section{Microdialysis Probe Calibration In vitro Calibration}

Microdialysis probe recovery rates were evaluated in vitro and in vivo. The objective of the in vitro calibration study was to determine the diffusion characteristics of MTX at different concentrations by the CMA30 dialysis probe. For this, each linear probe $(2 \mu \mathrm{L} / \mathrm{min}$ flow rate, $\mathrm{n}=3)$ was exposed to various concentrations of $\operatorname{MTX}(1,10$, and $100 \mu \mathrm{M})$ at $37^{\circ} \mathrm{C}$ with gentle agitation. Samples from the probe outlet were collected every $10 \mathrm{~min}(20 \mu \mathrm{L})$ for $2 \mathrm{~h}$ and the resulting concentration of MTX was determined. Subsequently, the relative recoveries were calculated by determining the ratio of the probe outlet concentration of MTX to the concentration of the external solution of MTX in the proximity of the probe, and expressing the ratio as a percentage $\left(\mathrm{C}_{\text {out }}\right.$ $\left./ \mathrm{C}_{\text {medium }}\right) \times 100$. Additionally, the reaction time of the probe was determined using sequential replacement 
of the surrounding medium with different concentrations of MTX and water at the end of each cycle.

\section{In vivo Calibration}

Relative recovery (RR) of MTX in vivo was evaluated using the retrodialysis method. In retrodialysis, or reverse dialysis, the drug itself is added to the perfusate fluid and its in vivo loss is used as a measure of in vivo recovery. The principle of this method relies on the assumption that the diffusion process is quantitatively equal in both directions across the semi-permeable membrane. Therefore, the relative loss of the drug into the tissue, before the experiment, is representative of its recovery, or relative gain, from the tissue during the experiment. Thus, based on this principle, known concentrations of MTX solution were included in the perfusate fluid and the rate of drug disappearance through the membrane was subsequently calculated as the in vivo recovery. $\mathrm{C}_{\text {out }}$ (MTX concentration in the dialysate) was measured and RR (\%) was calculated as the in vivo recovery according to the following equation:

$$
\operatorname{RR}(\%)=100-\left[100 \bullet\left(\mathrm{C}_{\text {dialysate }} / \mathrm{C}_{\text {perfusate }}\right)\right]
$$

Animals (10-14 weeks old) were placed on their ventral surface and the introducer needle was inserted initially through the skin and positioned between the skin and the muscle where it was withdrawn after the probe membrane was placed subcutaneously. The dialysis membrane was positioned in the center of the two insertion sites, and kept at the site using surgical tape. The in vivo recovery assessment based on the retrodialysis method was performed similar to the in vitro calibration using three different concentrations of $\operatorname{MTX}(1,10$, and $100 \mu \mathrm{M})$ in xenografted nude mice (3 animals/concentration).

\section{Corrections for Microdialysis Experiments}

The concentrations of MTX in the dialysate samples obtained in microdialysis experiments were corrected based on the in vivo recovery of the proximate dialysate concentration obtained from each recovery study. Estimates of the "true" dialysate concentrations were calculated based on the in vivo relative recovery by the following equation:

\footnotetext{
"True" dialysate concentration $=100 \bullet$ dialysate concentration / in vivo recovery value
}

\section{Data Collection}

The animal work was approved by IACUC of Charles River Laboratories, Shrewsbury, MA. Details of experimental procedures have been described in our previous investigation $(22,23)$. Briefly, $200 \mathrm{mg} / \mathrm{kg}$ MTX was intravenously injected into two groups of mice (CD-1 or nude strain). The first group (CD-1 mice) was used to collect plasma at $1,3,5,15,30,60$ and $120 \mathrm{~min}$ after drug administration. The second group included nude mice subcutaneously xenografted (a size of $600 \mathrm{mg}$ ) with breast cancer (MDA-MB-231) tumor. Throughout the study, the length (L) and width (W) of any tumors that developed were measured in millimeters using calibrated vernier calipers, where $\mathrm{L}$ is the longer of the two dimensions. The corresponding tumor weight was calculated by using the following experimental formula: Weight of the tumor $(\mathrm{mg})=\left(\mathrm{L} \mathrm{x} \mathrm{W}^{2}\right) / 2$. The core of the tumor was visually defined as a half of the measured length of the tumor. Microdialysis probes $(2 \mu \mathrm{L} / \mathrm{min}$ flow rate) were used to collect the unbound drug after the equilibrium from the core of the tumor at time periods of $10 \mathrm{~min}$ to $120 \mathrm{~min}$ with an interval of every $10 \min (n=3)$. The HPLC method was used to quantify MTX concentrations from collected samples using the method described in our previous publication (23). All the raw data, instead of the mean values, were used in the pharmacokinetic analysis based on the pharmacokinetic model introduced below.

\section{Hybrid physiologically-based pharmacokinetic modeling of MTX tumor transport}

A hybrid physiologically-based pharmacokinetic (hPBPK) model was designed to focus on a single target of interest, such as tumor, to avoid the complexities and considerable resources of developing a global or whole animal physiologic model (24). There are two constituents of a hybrid pharmacokinetic model, 1) a forcing function; which is an equation describing plasma drug concentrations profile, and 2) a model for a tissue or tumor compartment that can retain the physiologic attributes, such as organ blood flow (24-27). The condensed nature of the hybrid PK model requires drug concentration measurements in at least two sites, plasma and tumor. Briefly, to convert total MTX concentrations to free (unbound) MTX concentrations in plasma the measured plasma concentrations were factored by 0.6 , a degree of plasma protein binding of MTX (28). This 
conversion was necessary since the measured MTX concentrations in the tumor by microdialysis only reflect the free concentrations of the drug. An assumption was made that protein binding of MTX during the experiment period would remain constant. The free drug concentration-time profile of plasma MTX was described by a biexponential function $\left(\mathrm{A} \cdot \mathrm{e}^{-\alpha \bullet t}+\mathrm{B} \cdot \mathrm{e}^{-\beta \bullet t}\right)$ using SAAM $\mathrm{II}^{\circledR}$ (version 2.2, University of Washington, Seattle, WA, USA). Then, the forcing function, $f(t)$, was obtained by multiplying the biexponential equation by the physiologic blood flow rate $(Q)$ to the tumor:

$$
f(t)=\left(\mathrm{A} \cdot \mathrm{e}^{-\alpha \cdot t}+\mathrm{B} \cdot \mathrm{e}^{-\beta \cdot t}\right) \cdot \mathrm{Q}
$$

The forcing function was subsequently used as an input function into the tumor "core". A tumor core was subdivided into vascular $(V)$ and interstitial spaces (I) (Figure 1-A). This approach of dividing tumor into different spaces was previously employed by Gallo et al. to build an hPBPK model for temozolomide (24). In addition, we introduced a lag time ( $\left.T_{\text {lag }}\right)$ to account for a delayed appearance of the drug in the tumor. The $\mathrm{T}_{\text {lag }}$ was estimated by a curve feathering method and subtracted from the time profile of the tumor concentrations. In order to increase parameter identifiability, the relative "volume" ratio $(r)$ of tumor interstitial fluid volume $\left(V_{i}\right)$ to vascular volume $\left(V_{v}\right)$ was obtained from the literature (24). This $r$ value was used in the model to reduce the number of unknown parameters. We assumed $C L_{v i}=C L_{i v}$, and thereby $j_{v i} \bullet V_{v}=j_{i v} \bullet V_{i}$ (Figure. 1-A). Since $V_{i} / V_{v}=r$, and $j_{i v}=j_{v i} /$ r, the model is left with only three unknown parameters $\left(j_{v i}, j_{i 0}\right.$ and $V_{v}$ ), which are solved by modeling analysis (see Table 1 for symbol description).

\section{Sensitivity Analysis}

In order to understand the impact of pharmacokinetic parameters on the tumor disposition of MTX, the sensitivity of the hPBPK model parameters was examined by perturbing the values of each model parameter by several orders of magnitude (i.e. 0.125 , $0.25,0.5,1,2,4$ and 8 folds). The concentration-time profiles of MTX were subsequently simulated using the altered values and the impact of parameters alteration on the MTX tumor concentration profiles, AUC and mean residence time (MRT) was evaluated. The AUC was calculated by the trapezoidal method with an extrapolation from the last time point to time infinity. The MRT was computed from dividing the area under the first moment curve (AUMC) by AUC.

\section{Prediction of MTX concentrations in the human tumor using the hPBPK model}

In order to describe MTX disposition in human tumors, we attempted to scale up our hPBPK mouse model. The relevant physiological PK parameters from the mouse hPBPK model, including plasma flow rate $(Q)$ and the rate constants $\left(\mathrm{j}_{\mathrm{vi}}\right.$ and $\left.\mathrm{j}_{\mathrm{i} i}\right)$, were scaled up using the following equation:

Human parameter $=$ Mouse parameter $\bullet($ Human body weight/Mouse body weight $)^{\mathrm{b}}$; where $\mathrm{b}$ is an allometric exponent fixed to 0.75 and 0.25 for the scaling of $Q$ and $j_{v i} / j_{i 0}$ parameters, respectively. The total tumor volume was estimated to be $65.4 \mathrm{~mL}$ based on the tumor diameter of $5 \mathrm{~cm}$ from the human MTX data in the literature (29). Since the interstitial volume of the breast tumor is $50 \%$ of the tumor size (30), the interstitial tumor volume was estimated to be $32.7 \mathrm{~mL}$ in humans. Once the PK parameters were scaled up, the human plasma and tumor data for MTX were utilized from the literature (29). Subsequently, the forcing function equation was recalculated using the free MTX plasma concentrations in humans $(50 \%$ protein binding was taken into the consideration (31)) and incorporated into the hPBPK model. The final scaled-up PK parameters which were used to simulate human MTX tumor concentrations are provided in Table 2.

\section{RESULTS}

\section{In vitro Calibration}

In vitro recovery experiments demonstrated that a stable equilibrium was attained after $30 \mathrm{~min}$, and that the relative recovery of MTX after equilibration was constant at three different concentrations: 1, 10, and $100 \mu \mathrm{M}$ (Figure. 1). The mean in vitro recovery for MTX was $39.3 \pm 3.5 \%$. The reaction time of the linear probe, when MTX concentration in the surrounding medium was changed, was $20 \mathrm{~min}$ and the probe was cleared of the drug within 20 min after MTX solutions were replaced with water. 


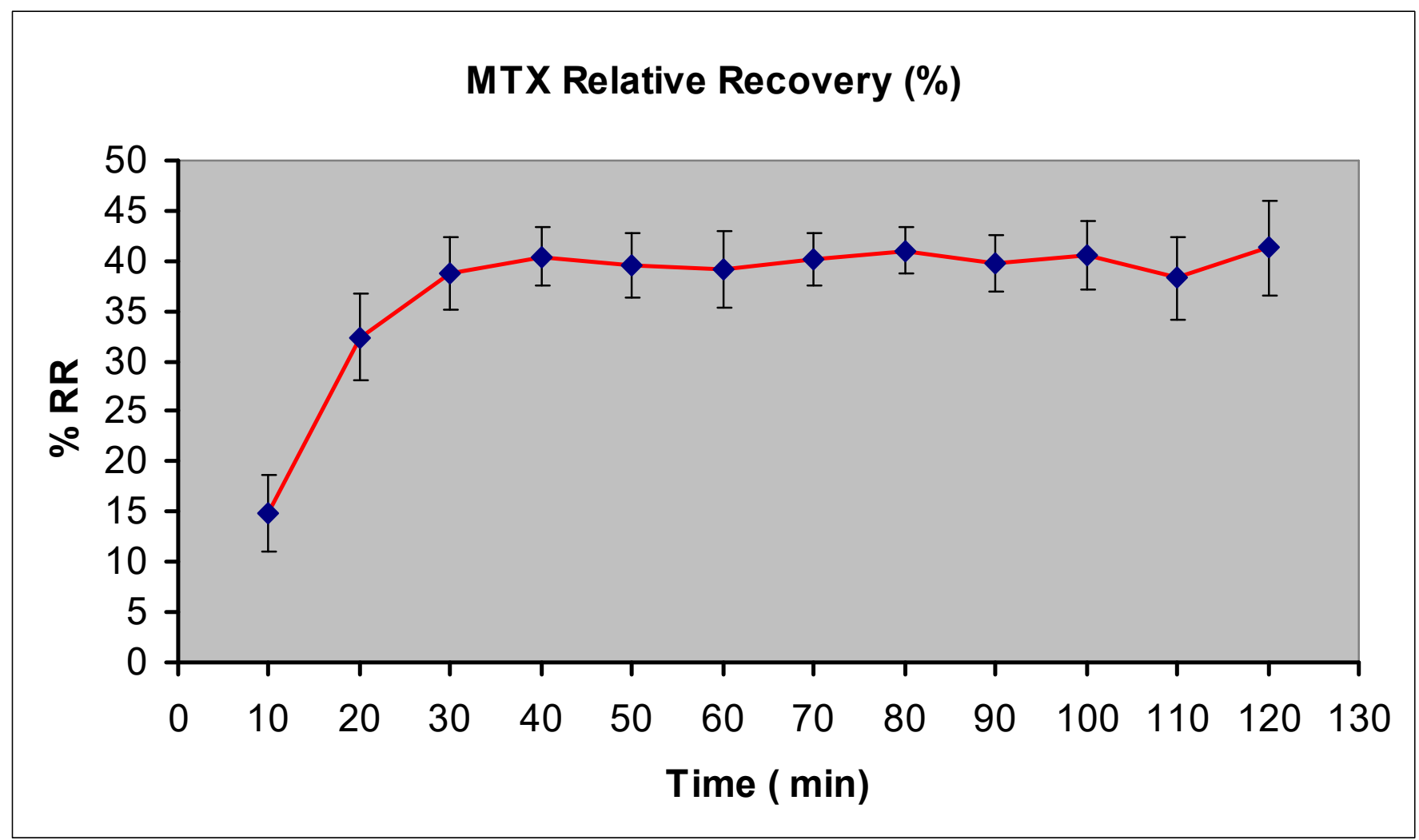

Figure 1. In vitro relative recovery (\%) of MTX. Time-dependent equilibration of MTX $(100 \mu \mathrm{M})$ during in vitro microdialysis. Microdialysis was performed at $37^{\circ} \mathrm{C}$ with gentle agitation. Data are expressed as mean $\pm \mathrm{SE}(\mathrm{n}=3)$.

\section{In vivo Calibration}

In vivo data indicated that equilibrium conditions in nude mice were reached after $40 \mathrm{~min}$ and that recovery was concentration-independent. The mean in vivo recovery for all concentrations of MTX was $25.5 \pm 4.2 \%$.

\section{Hybrid physiologically-based pharmacokinetic modeling of MTX}

Prior to hPBPK model, the plasma concentrationtime profile was analyzed by a biexponential decay model in order to obtain a forcing function that enters the vascular space in the tumor compartment (Figure. 2-A).

The plasma concentration of MTX (Figure. 2-B) was described as:

$\mathrm{C}_{\mathrm{p}}=896.1 \cdot \mathrm{e}^{-0.485 \cdot \mathrm{t}}+35.5 \cdot \mathrm{e}^{-0.022 \cdot \mathrm{t}}$
The associated rate constants and volumes of distribution are shown in Table 1. The forcing function, a product of $\mathrm{C}_{\mathrm{p}}$ and plasma flow into the tumor $(0.1065 \mathrm{~mL} / \mathrm{min})$, was subsequently defined as:

$f(t)=\left(896.1 \cdot \mathrm{e}^{-0.485 \cdot \mathrm{t}}+35.5 \cdot \mathrm{e}^{-0.022 \cdot \mathrm{t}}\right) \cdot 0.1065$

Using the forcing function $f(t)$, the tumor free concentrations of MTX were fitted to the hPBPK model with a focus on tumor vascular and interstitial spaces. The rate constant characterizing MTX transfer from the vascular to the interstitial space $\left(j_{v i}\right)$ was estimated to be $0.0233 \mathrm{~min}^{-1}$ whereas the transfer rate constant from the interstitial space to the vascular space $\left(j_{i v}\right)$ was $0.00602 \mathrm{~min}^{-1}$. This model also provided an exit rate constant of the drug from the tumor through the interstitial space $\left(j_{i 0}=0.286\right.$ $\left.\min ^{-1}\right)$. The model parameters are summarized in Table 1. 

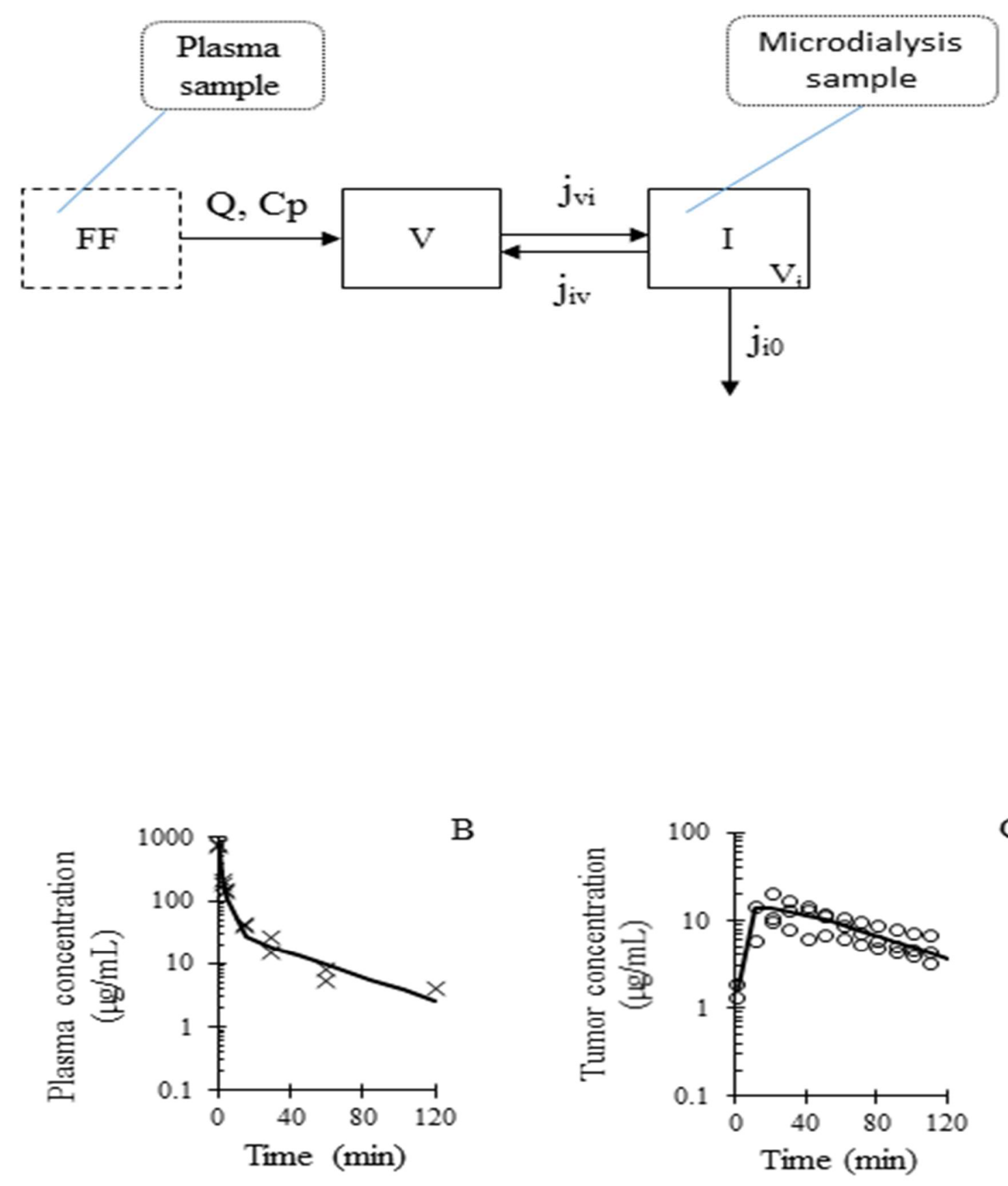

$\mathrm{C}$

Figure 2. Hybrid physiologically-based pharmacokinetic (hPBPK) model of MTX. A: Schematic structures of hPBPK model for MTX disposition. On the left is the plasma part, which is the source of the driving force of a biexponential decay function $\left(C_{p}\right)$ that is used as an input into the tumor. On the right is the tumor part which composes vascular and interstitial spaces. B: Plasma concentration-time profile of MTX after an IV bolus injection of the drug. Shown are measured MTX concentrations (x) and biexponential decay predicted concentrations of MTX (-). C: Tumor concentration-time profile of MTX after an IV bolus injection of the drug. Shown are measured MTX concentrations (o) and hPBPK model-predicted concentrations of MTX (-). 
Table 1. hPBPK model parameters.

\begin{tabular}{|c|c|c|}
\hline Parameter description (unit) & Symbol & Value (\%CV) \\
\hline Transfer rate constant from vascular space to interstitial space $\left(\mathrm{min}^{-1}\right)$ & $j_{v i}$ & $0.02334(9.0)$ \\
\hline Transfer rate constant from interstitial space to vascular space $\left(\mathrm{min}^{-1}\right)$ & $j_{i v}$ & $0.00602(9.0)$ \\
\hline Elimination rate constant from the tumor through the interstitial space $\left(\mathrm{min}^{-1}\right)$ & $j_{i 0}$ & $0.28602(21.0)$ \\
\hline Lag time (min) * & $T_{\text {lag }}$ & 8.5 \\
\hline The relative ratio of tumor interstitial fluid volume to vascular volume & $r$ & $3.875[$ fixed, $(32)]$ \\
\hline Interstitial volume of tumor (mL) & $V_{i}$ & $1.03327(21.0)$ \\
\hline First term constant in the forcing function equation $(\mu \mathrm{g} / \mathrm{mL})$ & $A$ & $896.1(74.6)$ \\
\hline Second term constant in the forcing function equation $(\mu \mathrm{g} / \mathrm{mL})$ & $B$ & $35.5(54.0)$ \\
\hline Disposition rate constant of the first term in the forcing function equation $\left(\mathrm{min}^{-1}\right)$ & $\alpha$ & $0.485(51.5)$ \\
\hline Disposition rate constant of the second term in the forcing function equation $\left(\mathrm{min}^{-1}\right)$ & $\beta$ & $0.022(39.7)$ \\
\hline Plasma flow rate $(\mathrm{mL} / \mathrm{min})$ & $Q$ & 0.1065 [fixed, (32)] \\
\hline Area under the curve in tumor $(\min \bullet \mu \mathrm{g} / \mathrm{mL})$ & Tumor $A U C$ & 1313.2 \\
\hline Mean residence time in tumor (min) & Tumor $M R T$ & 107.4 \\
\hline Total body clearance $(\mathrm{mL} / \mathrm{min} \bullet \mathrm{kg}) * *$ & $C L$ & 47.0 \\
\hline \multicolumn{3}{|c|}{$\begin{array}{l}* \text { Estimated by curve feathering method. } \\
* * \text { Obtained from the forcing function. } \% \mathrm{CV} \text {, the percent of the coefficient of variation }(\mathrm{CV}) . \mathrm{V}_{\mathrm{i}}, \mathrm{A}, \mathrm{B}, \mathrm{AUC} \text { and } \mathrm{CL} \text { values } \\
\text { are based on the free MTX concentrations. }\end{array}$} \\
\hline
\end{tabular}

Sensitivity analysis. Sensitivity analysis of the hPBPK model showed that $j_{v i}$ was the most effective parameter on changing the terminal slope of the tumor profile (Figure. 3). A reduction in $j_{v i}$ by 8-time resulted in an increased MRT by 5.7 folds (Figure. 3 ). However, a change in $j_{v i}$ value did not influence the AUC of MTX in tumor interstitium (Figure. 4). An 8-fold perturbation of $j_{i v}$ did not affect the terminal slope, AUC or MRT of MTX in the tumor (Figures. 3 and 4). Additionally, decreased $j_{i 0}$ and $V_{i}$ values did not alter the terminal slopes of MTX, but they increased AUC values with a negligible effect on MRT of MTX in the tumor (Figures. 3 and 4). Finally, a perturbation of $Q$ resulted in a direct effect on AUC value without affecting the terminal slopes or MRT of MTX in tumor (Figures. 3 and 4).

\section{hPBPK model-based simulation of MTX concentrations in human tumor}

We further examined if our pre-clinical hPBPK model can reasonably predict the disposition of MTX in human tumor. The previously published human MTX concentrations from both plasma and tumor microdialysis (29) were utilized in this exercise. The simulation was conducted as described previously in the Materials and Methods section. The results shown in Figure 5 indicated that our model recapitulated MTX concentrations in human breast tumor. Table 2 shows pharmacokinetic parameters associated with tumor transport of MTX in humans.

\section{DISCUSSION}

To gain therapeutic benefits, a drug must reach the site of action at adequate concentrations. Traditional pharmacokinetic models utilize plasma concentrations as a surrogate marker that reflects drug concentrations at the site of action. However, in many cases plasma concentrations alone have failed to estimate drug concentrations in tissues of interest (15-17). Microdialysis is a sampling technique that allows the measurements of free drug concentrations within the target tissues. Microdialysis was used to obtain the concentration-time profile of MTX, our drug of interest, at the tumor site. We chose MTX for several reasons; MTX has played a crucial role in cancer chemotherapy in combination with other chemotherapeutic agents in the treatment of various human malignancies, including breast cancer, osteosarcoma, and lung cancer $(14,33)$. The MTX dose used in this experiment $(200 \mathrm{mg} / \mathrm{kg})$ in mice is equivalent to $600 \mathrm{mg} / \mathrm{m}^{2}$ (34) which falls into a lowdose MTX regimen (35). The systemic PK of MTX is well-characterized and a large body of data is available in the literature $(36,37)$. 


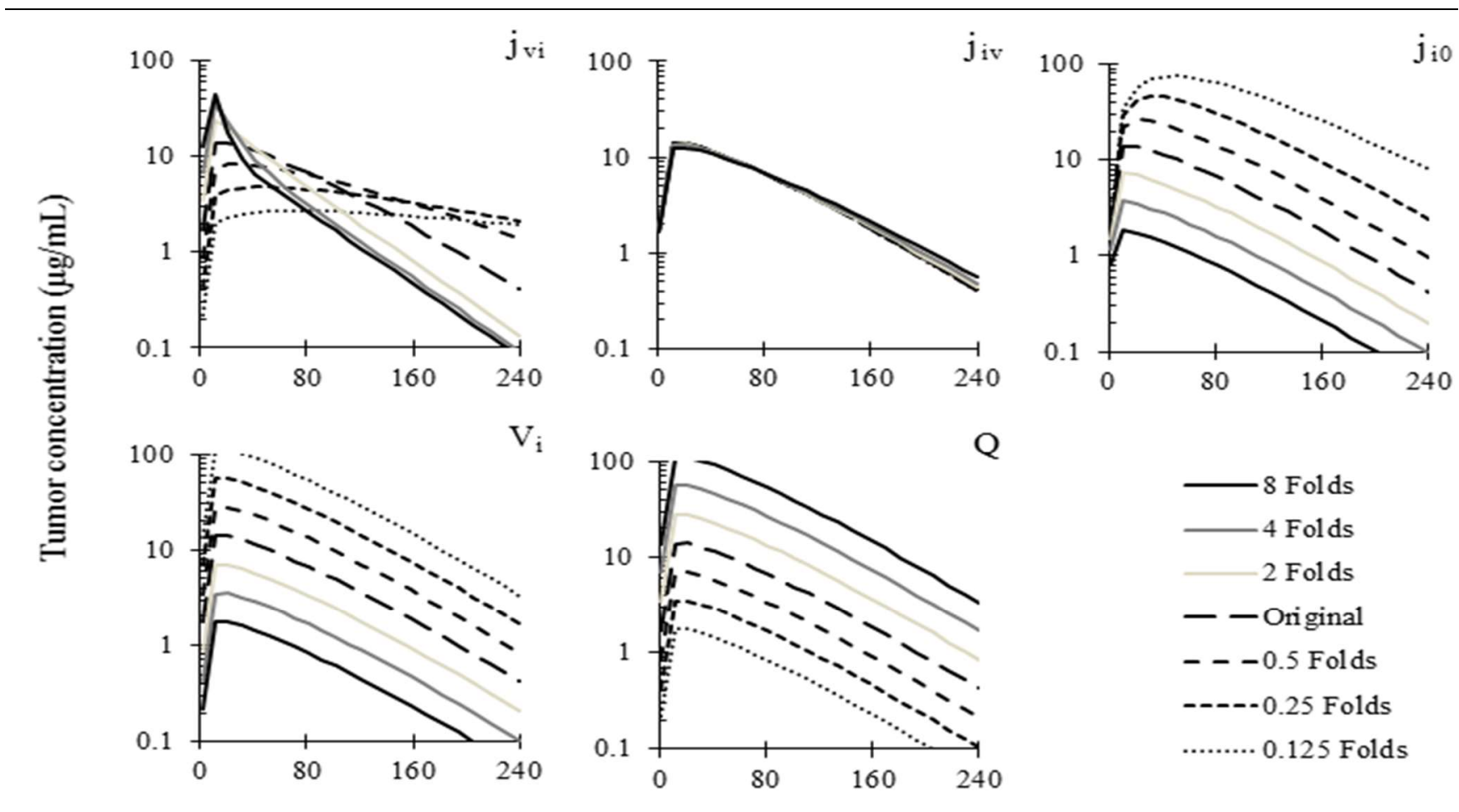

Time (min)

Figure 3. Simulated MTX tumor concentrations with changes in PK parameters of the hPBPK model. To better understand the influence of pharmacokinetic parameters on the tumor disposition of MTX, PK parameter values were altered by several folds above and below original values and MTX concentration-time profiles in the tumor were characterized.

However, little is known about its accumulation in tumors and normal tissues. Moreover, preliminary clinical evidence has shown that plasma levels of MTX are not predictive of intra-tumor levels and there is a high inter-subject variability in clinical response to $\operatorname{MTX}(29,36)$. This deficiency provided us with the impetus to develop a more comprehensive pre-clinical PK model of MTX in breast tumors that could subsequently be scaled to predict human breast MTX concentrations. In this study, MTX concentration-time profiles that we previously obtained from plasma and tumor $(22,23)$ were used to develop a hPBPK model to better describe MTX disposition in solid tumor and to more precisely predict MTX concentrations in the tumor.

Although the compartment model can quantitatively describe MTX disposition in both plasma and tumor, it does not represent real physical spaces or physiological processes in the body. In contrast, hPBPK model offers better physiological understanding of the model, such as tumor plasma flow and physical volume of tumor interstitial fluid. This additional information can facilitate allometric scaling from animal models to humans. The hPBPK model is more focused on MTX disposition within tumor regions than the conventional whole-body PBPK. Instead of using a transit compartment to account for the delayed appearance of MTX in the tumor, $\mathrm{T}_{\text {lag }}$ was introduced to the model to allow for curve fitting and estimation of PK parameters. This approach of using $T_{\text {lag }}$ reduced the number of unknown parameters and permitted the use of a simpler model. Since the hPBPK model is focused on MTX disposition in the tumor with a fixed input forcing function from plasma, the sensitivity analysis was conducted only for tumor spaces. Our model demonstrated that the effect of $j_{v i}$ on the terminal slope and MRT of MTX in the tumor is significant. In addition, a perturbation in $j_{v i}$ did not affect the AUC value. One can find that $j_{v i}$ describes the entry rate of distribution to the sampling site (tumor). Our hPBPK model also suggests that a reduction in $j_{i 0}$ and $j_{v i}$ will provide the best therapeutic strategy since the AUC and MRT of MTX will increase in the tumor (Figure. 3). 

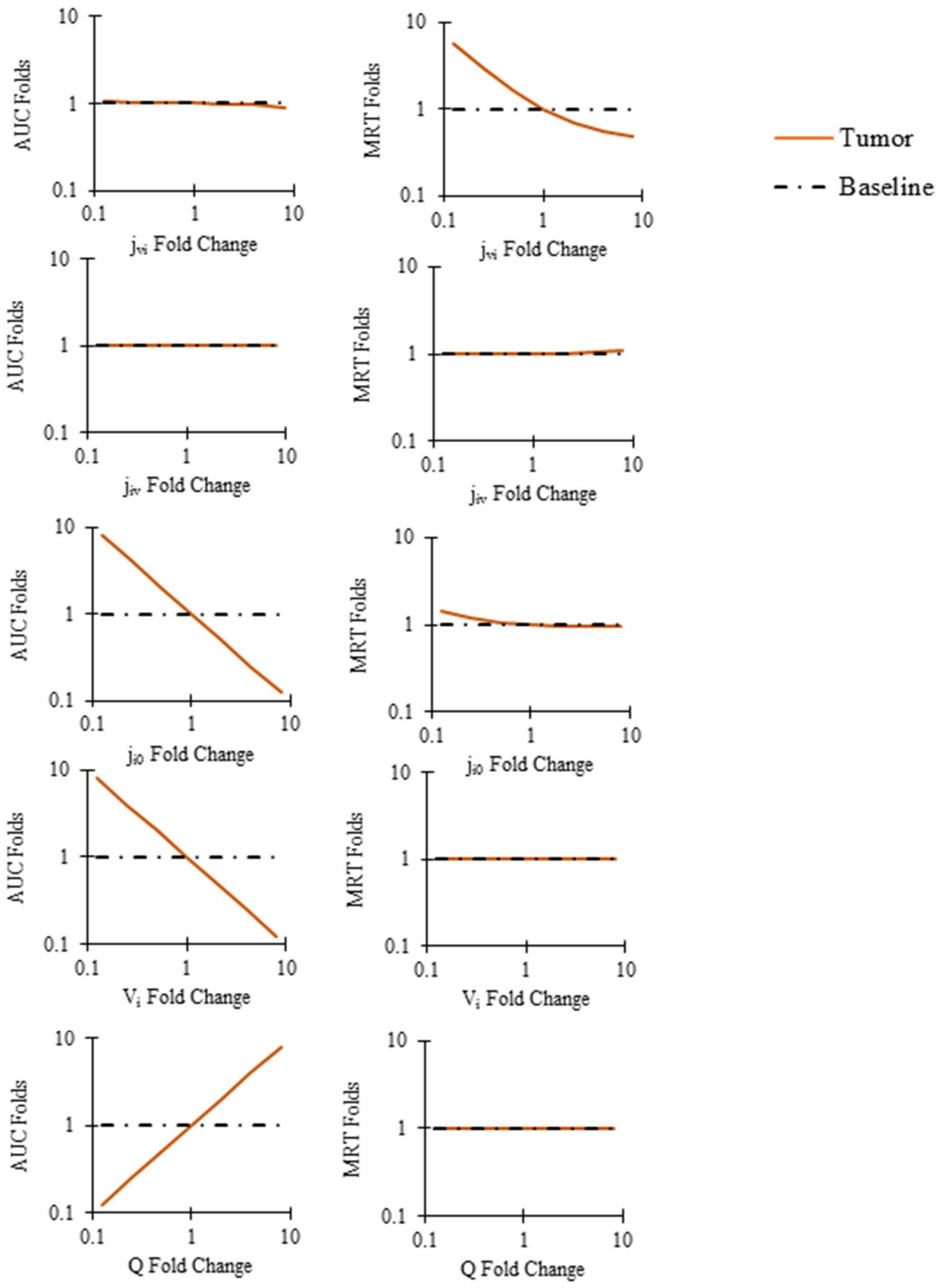

Figure 4. Sensitivity analysis on AUC and MRT upon changes in PK parameters of the hPBPK model. To identify the effect of certain PK parameters on AUC and MRT, PK parameter value of interest was changed while fixing the rest of the parameters during simulation process. Subsequently, AUC and MRT were calculated using the trapezoidal method utilizing the simulated concentration time profile. 
It is plausible to reduce the rate constant, $j_{i 0}$, by either formulating novel nano-particulate delivery systems of MTX, or by conjugated forms of MTX and/or by modifying physicochemical properties of such drug delivery systems. In addition, our model can help in drug combination studies where one can enhance
MTX accumulation in the tumor. For instance, the rate constants of MTX in the tumor could be altered by transporter modulators (e.g. Pgp inhibitors) in the tumor environment to increase MTX efficacy, which can be studied using animal models along with the hPBPK model.

Table 2. Scaled-up hPBPK parameters from mouse to human.

\begin{tabular}{|c|c|c|}
\hline Parameter description (unit) & Symbol & Value (\%CV) \\
\hline Transfer rate constant from vascular space to interstitial space $\left(\mathrm{min}^{-1}\right)$ & $j_{v i}$ & 0.151 (scaled-up) \\
\hline Transfer rate constant from interstitial space to vascular space $\left(\mathrm{min}^{-1}\right)$ & $j_{i v}$ & 0.03897 (scaled-up) \\
\hline Elimination rate constant from the tumor through the interstitial space $\left(\mathrm{min}^{-1}\right)$ & $j_{i 0}$ & 1.85 (scaled-up) \\
\hline Lag time $(\mathrm{min}) *$ & $T_{\text {lag }}$ & 25 \\
\hline The relative ratio of tumor interstitial fluid volume to vascular volume & $r$ & 3.875 [fixed, (32)] \\
\hline Interstitial volume of tumor (mL) & $V_{i}$ & 32.7 (scaled-up) \\
\hline First term constant in the forcing function equation $(\mu \mathrm{g} / \mathrm{mL})$ & $A$ & $4.34(12.0)$ \\
\hline Second term constant in the forcing function equation $(\mu \mathrm{g} / \mathrm{mL})$ & $B$ & $0.72(10.4)$ \\
\hline Disposition rate constant of the first term in the forcing function equation $\left(\mathrm{min}^{-1}\right)$ & $\alpha$ & $0.055(11.0)$ \\
\hline Disposition rate constant of the second term in the forcing function equation $\left(\mathrm{min}^{-1}\right)$ & $\beta$ & $0.0019(38.6)$ \\
\hline Plasma flow rate $(\mathrm{mL} / \mathrm{min})$ & $Q$ & 28.8 (scaled-up) \\
\hline Area under the curve in tumor $(\min \bullet \mu \mathrm{g} / \mathrm{mL})$ & Tumor $A U C$ & 69.9 \\
\hline Mean residence time in tumor (min) & Tumor $M R T$ & 106.5 \\
\hline Total body clearance $(\mathrm{mL} / \mathrm{min} \bullet \mathrm{kg}) * *$ & $C L$ & 2.6 \\
\hline \multicolumn{3}{|c|}{$\begin{array}{l}\text { * Estimated by visual inspection since the rising phase in tumor concentrations was not captured in human data from the } \\
\text { literature. ** Obtained from the forcing function. } \% \mathrm{CV} \text {, the percent of the coefficient of variation }(\mathrm{CV}) \text {. } \\
\mathrm{V}_{\mathrm{i}}, \mathrm{A}, \mathrm{B}, \mathrm{AUC} \text { and CL values are based on the free MTX concentrations. }\end{array}$} \\
\hline
\end{tabular}

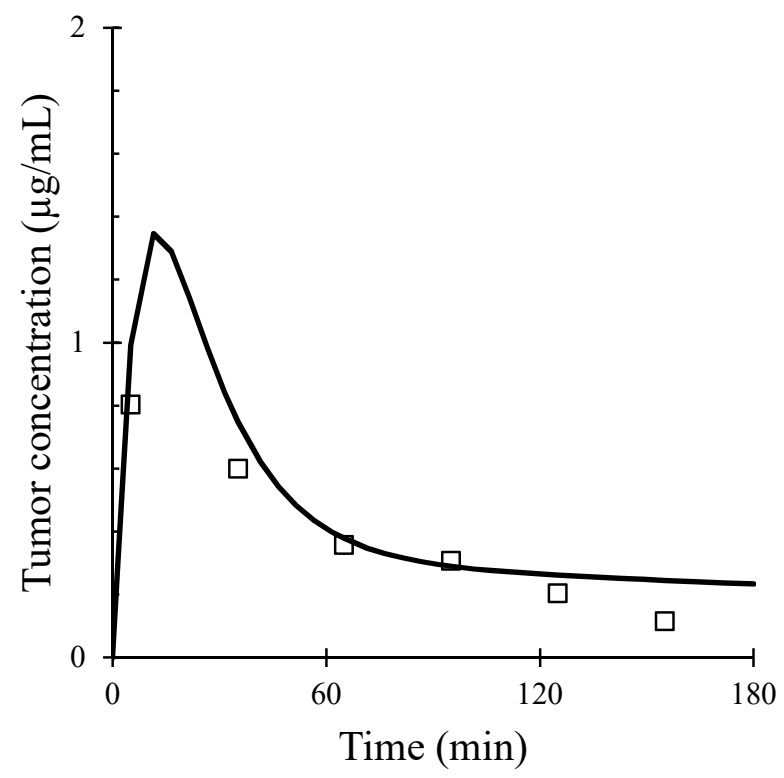

Figure 5. Simulation of MTX concentrations in human breast tumor using the scaled-up hPBPK model. Open squares are observed data from human MTX tumor-microdialysis and the solid line represents predicted MTX concentrations in human breast tumor using the scaled-up hPBPK model. 
The utility of our hPBPK model was validated using published MTX data from human subjects with breast cancer. Physiological PK parameters were utilized for the inter-species scale-up which ultimately allowed us to simulate MTX concentrations in the human tumor. We noted that the simulation profile did not accurately capture the measured concentrations at the last time point. This discrepancy could be a result of the different mechanism of MTX transport at various concentrations. At high concentrations as in our mouse experiment, MTX follows passive diffusion, whereas lower MTX concentrations in human studies may follow active saturable transport, as discussed in the following section.

Although our hPBPK model in this investigation was able to quantitatively describe MTX disposition based on MTX concentrations collected from both plasma and tumor, there are several drawbacks in this model that may affect the accuracy of predictions. First, MTX may follow both linear and/or nonlinear kinetics depending on the available free concentrations of MTX at the site of transport (38). At low concentrations, MTX is transported by $\mathrm{ABC}$ transporters and organic anion transporters (39, 40) as well as folate transporters that contribute to the nonlinear pharmacokinetic behavior of MTX. In this study, MTX transport may include both linear and nonlinear processes since MTX was IV bolus injected into the animal subjects. However, we did not incorporate the nonlinear process into our model due to the limitation of the single-dose experiment and the short duration of the experiment. Second, the plasma protein binding was assigned as a fixed value throughout the entire time profile. The element of the nonlinearity of MTX protein binding (41) was not taken into consideration in this model. In reality, any changes in the protein binding fraction may affect the prediction of the actual transfer rate constants. Third, the time frame for plasma MTX collection, which was within $120 \mathrm{~min}$, may be insufficient for a precise estimation of the terminal half-life or for gaining a complete picture of all phases of MTX disposition in plasma. However, a limited period of time is required for this experiment since mice needed to be anesthetized for the whole duration of the sampling period to ensure the accuracy of the position of the microdialysis probes. Fourth, whether or not the same transfer rate constant exists in normal tissues compared with the tumor tissues is questionable. Nevertheless, the difference in $\mathrm{pH}$ between the tumor and normal tissues is still considerable $(42,43)$. This difference in $\mathrm{pH}$ and resultant changes in transporter properties (44) may promote an asymmetrical transport of MTX into the tumor compared with normal tissues. In addition, microdialysis has its own limitations (45). While retrodialysis is a useful and simple calibration method commonly used to estimate recoveries, a drawback of this method is that possible changes in recovery over time are not measured (45-48). Recovery is influenced by various solute and tissue-related factors. Among these factors are the physico-chemical properties of the solute of interest and its diffusion coefficient in the tissue, the extracellular fluid volume fraction, and the processes for elimination from the tissue, including active transport mechanisms (46). Changes in recovery during the experiment can be partially taken into account via retrodialysis by calibrator and many of these factors have been incorporated as parameters in mathematical models (46-51). Finally, microdialysis only measures extracellular free concentrations rather than intracellular concentrations of drugs. This limitation could be overcome using a radiolabeled MTX that can give important information about the total amount of MTX in tumor, although MTX metabolites should also be adequately distinguished. Therefore, using complementary techniques will help to improve the proposed model by including intracellular concentrations of MTX in addition to extracellular ones obtained by microdialysis.

\section{CONCLUSIONS}

In summary, a hPBPK model was developed to better describe MTX disposition in the tumor based on free drug concentrations in tumor and plasma along with an incorporation of the physiologicallyrelevant values such as tumor blood flow and tumor interstitial volume. The hPBPK model helped to focus on MTX disposition in the tumor and allowed for the prediction of different scenarios upon perturbations of the PK parameters of the model. Furthermore, the model-based predictability of MTX concentrations in the tumor was verified using published human microdialysis data. Future studies should include more information about MTX concentrations from different regions of the tumor (i.e. core and periphery of the tumor) into the models, collecting MTX from non-human primate models (e.g. monkey species) and applying different transporter blockers to increase our understanding of MTX disposition in the tumor. 


\section{ACKNOWLEDGMENTS}

This work was supported in part by Jon Shevell Memorial Student Research Scholarship (H.H.A.) and Western New England University, College of Pharmacy (S.N.S.). The authors are grateful to Melody Chang for editorial assistance.

\section{CONFLICT OF INTEREST.}

The authors have no conflicting financial interests.

\section{REFERENCES}

1. Shargel L, Yu ABC. Applied biopharmaceutics \& pharmacokinetics2016.

2. Spruill WJ, Wade WE, DiPiro JT, Blouin RA, Pruemer JM, American Society of Health-System P. Concepts in clinical pharmacokinetics2014.

3. Borst P, Evers R, Kool M, Wijnholds J. A family of drug transporters: the multidrug resistance-associated proteins. J Natl Cancer Inst. 2000;92(16):1295-302.

4. Doyle L, Ross DD. Multidrug resistance mediated by the breast cancer resistance protein BCRP (ABCG2). Oncogene. 2003;22(47):7340-58.

5. Muller $M$, dela Pena A, Derendorf $H$. Issues in pharmacokinetics and pharmacodynamics of antiinfective agents: distribution in tissue. Antimicrob Agents Chemother. 2004;48(5):1441-53.

6. Muller M, Mader RM, Steiner B, Steger GG, Jansen $\mathrm{B}$, Gnant $\mathrm{M}$, et al. 5-fluorouracil kinetics in the interstitial tumor space: clinical response in breast cancer patients. Cancer Res. 1997;57(13):2598-601.

7. Presant CA, Wolf W, Waluch V, Wiseman C, Kennedy P, Blayney D, et al. Association of intratumoral pharmacokinetics of fluorouracil with clinical response. Lancet. 1994;343(8907):1184-7.

8. Gelmon KA, Eisenhauer EA, Harris AL, Ratain MJ, Workman P. Anticancer agents targeting signaling molecules and cancer cell environment: challenges for drug development? J Natl Cancer Inst. 1999;91(15):1281-7.

9. Kamb A. What's wrong with our cancer models? Nat Rev Drug Discov. 2005;4(2):161-5.

10. Hryniuk WM. The importance of dose intensity in the outcome of chemotherapy. Important Adv Oncol. 1988:121-41.

11. Jain RK. Delivery of novel therapeutic agents in tumors: physiological barriers and strategies. J Natl Cancer Inst. 1989;81(8):570-6.

12. Jain RK. Vascular and interstitial barriers to delivery of therapeutic agents in tumors. Cancer Metastasis Rev. 1990;9(3):253-66.

13. Workman P, Graham MA, Imperial Cancer Research F. Pharmacokinetics and cancer chemotherapy.
Plainview, N.Y.: Cold Spring Harbor Laboratory Press; 1993.

14. Micromedex I. Micromedex healthcare series.

15. Ekstrom PO, Andersen A, Saeter G, Giercksky KE, Slordal L. Continuous intratumoral microdialysis during high-dose methotrexate therapy in a patient with malignant fibrous histiocytoma of the femur: a case report. Cancer Chemother Pharmacol. 1997;39(3):267-72.

16. Ekstrom PO, Giercksky KE, Andersen A, Bruland OS, Slordal L. Intratumoral differences in methotrexate levels within human osteosarcoma xenografts studied by microdialysis. Life Sci. 1997;61(19):P1275-80.

17. Saeter G, Alvegard TA, Elomaa I, Stenwig AE, Holmstrom T, Solheim OP. Treatment of osteosarcoma of the extremities with the T-10 protocol, with emphasis on the effects of preoperative chemotherapy with single-agent high-dose methotrexate: a Scandinavian Sarcoma Group study. J Clin Oncol. 1991;9(10):1766-75.

18. Chu J, Gallo JM. Application of microdialysis to characterize drug disposition in tumors. Adv Drug Deliv Rev. 2000;45(2-3):243-53.

19. Westerhout J, van den Berg DJ, Hartman R, Danhof $\mathrm{M}$, de Lange EC. Prediction of methotrexate CNS distribution in different species - influence of disease conditions. Eur J Pharm Sci. 2014;57:11-24.

20. Bischoff KB, Dedrick RL, Zaharko DS, Longstreth JA. Methotrexate pharmacokinetics. J Pharm Sci. 1971;60(8):1128-33.

21. Simon N, Marsot A, Villard E, Choquet S, Khe HX, Zahr N, et al. Impact of ABCC2 polymorphisms on high-dose methotrexate pharmacokinetics in patients with lymphoid malignancy. Pharmacogenomics J. 2013;13(6):507-13.

22. Sani SN. Application of microdialysis to characterize drug disposition in pharmacokinetic studies : the case of methotrexate 2009.

23. Sani SN, Henry K, Bohlke M, Kim J, StrickerKrongrad A, Maher TJ. The effects of drug transporter inhibitors on the pharmacokinetics and tissue distribution of methotrexate in normal and tumor-bearing mice: a microdialysis study. Cancer Chemother Pharmacol. 2010;66(1):159-69.

24. Gallo JM, Vicini P, Orlansky A, Li S, Zhou F, Ma J, et al. Pharmacokinetic model-predicted anticancer drug concentrations in human tumors. Clin Cancer Res. 2004;10(23):8048-58.

25. Gallo JM, Etse JT, Doshi KJ, Boudinot FD, Chu CK. Hybrid pharmacokinetic models to describe anti-HIV nucleoside brain disposition following parent and prodrug administration in mice. Pharm Res. 1991;8(2):247-53.

26. Savic RM, Jonker DM, Kerbusch T, Karlsson MO. Implementation of a transit compartment model for describing drug absorption in pharmacokinetic 
studies. J Pharmacokinet Pharmacodyn. 2007;34(5):711-26.

27. Wada DR, Ward DS. The hybrid model: a new pharmacokinetic model for computer-controlled infusion pumps. IEEE Trans Biomed Eng. 1994;41(2):134-42.

28. Ekstrøm P, Andersen A, Warren D, Giercksky K, Slørdal L. Pharmacokinetics of different doses of methotrexate at steady state by in situ microdialysis in a rat model. Cancer Chemother Pharmacol. 1995;36(4):283-9.

29. Muller M, Brunner M, Schmid R, Mader RM, Bockenheimer J, Steger GG, et al. Interstitial methotrexate kinetics in primary breast cancer lesions. Cancer Res. 1998;58(14):2982-5.

30. Jain RK. Transport of molecules in the tumor interstitium: a review. Cancer Res. 1987;47(12):3039-51.

31. Thomson.Micromedex. Drug Information for the Health Care Professional. 24th ed. Content Reviewed by the United States Pharmacopeial Convention, Inc Greenwood Village, CO. 2004; Volume 1:1912.

32. Gallo JM, Vicini P, Orlansky A, Li S, Zhou F, Ma J, et al. Pharmacokinetic Model-Predicted Anticancer Drug Concentrations in Human Tumors. Clin Cancer Res. 2004;10(23):8048-58.

33. Purcell WT, Ettinger DS. Novel antifolate drugs. Curr Oncol Rep. 2003;5(2):114-25.

34. Freireich EJ, Gehan EA, Rall DP, Schmidt LH, Skipper HE. Quantitative comparison of toxicity of anticancer agents in mouse, rat, hamster, dog, monkey, and man. Cancer Chemother Rep. 1966;50(4):219-44.

35. Treon SP, Chabner BA. Concepts in use of high-dose methotrexate therapy. Clin Chem. 1996;42(8 Pt 2):1322-9.

36. Bleyer WA. The clinical pharmacology of methotrexate: new applications of an old drug. Cancer. 1978;41(1):36-51.

37. Shen DD, Azarnoff DL. Clinical pharmacokinetics of methotrexate. Clin Pharmacokinet. 1978;3(1):1-13.

38. de Graaf D, Sharma RC, Mechetner EB, Schimke RT, Roninson IB. P-glycoprotein confers methotrexate resistance in 3T6 cells with deficient carrier-mediated methotrexate uptake. Proc Natl Acad Sci U S A. 1996;93(3):1238-42.

39. Vlaming ML, Pala Z, van Esch A, Wagenaar E, van Tellingen $\mathrm{O}$, de Waart DR, et al. Impact of Abcc2 (Mrp2) and Abcc3 (Mrp3) on the in vivo elimination of methotrexate and its main toxic metabolite 7hydroxymethotrexate. Clin Cancer Res. 2008;14(24):8152-60.
40. Takeda M, Khamdang S, Narikawa S, Kimura H, Hosoyamada M, Cha SH, et al. Characterization of methotrexate transport and its drug interactions with human organic anion transporters. J Pharmacol Exp Ther. 2002;302(2):666-71.

41. Steele WH, Lawrence JR, Stuart JF, McNeill CA. The protein binding of methotrexate in the serum of patients with neoplastic disease. Cancer Chemother Pharmacol. 1981;7(1):61-4.

42. Poe M. Acidic dissociation constants of folic acid, dihydrofolic acid, and methotrexate. J Biol Chem. 1977;252(11):3724-8.

43. Tannock IF, Rotin D. Acid $\mathrm{pH}$ in tumors and its potential for therapeutic exploitation. Cancer Res. 1989;49(16):4373-84.

44. Breedveld P, Pluim D, Cipriani G, Dahlhaus F, van Eijndhoven MA, de Wolf CJ, et al. The effect of low $\mathrm{pH}$ on breast cancer resistance protein (ABCG2)mediated transport of methotrexate, 7hydroxymethotrexate, methotrexate diglutamate, folic acid, mitoxantrone, topotecan, and resveratrol in in vitro drug transport models. Mol Pharmacol. 2007;71(1):240-9.

45. de Lange EC, de Boer AG, Breimer DD. Methodological issues in microdialysis sampling for pharmacokinetic studies. Adv Drug Deliv Rev. 2000;45(2-3):125-48.

46. Chaurasia CS, Muller M, Bashaw ED, Benfeldt E, Bolinder J, Bullock R, et al. AAPS-FDA workshop white paper: microdialysis principles, application and regulatory perspectives. Pharm Res. 2007;24(5):1014-25.

47. Bungay PM, Dedrick RL, Fox E, Balis FM. Probe calibration in transient microdialysis in vivo. Pharm Res. 2001;18(3):361-6.

48. Bungay PM, Morrison PF, Dedrick RL. Steady-state theory for quantitative microdialysis of solutes and water in vivo and in vitro. Life Sci. 1990;46(2):10519.

49. Bungay PM, Sumbria RK, Bickel U. Unifying the mathematical modeling of in vivo and in vitro microdialysis. J Pharm Biomed Anal. 2011;55(1):5463.

50. Chefer VI, Zapata A, Shippenberg TS, Bungay PM. Quantitative no-net-flux microdialysis permits detection of increases and decreases in dopamine uptake in mouse nucleus accumbens. J Neurosci Methods. 2006;155(2):187-93.

51. Morrison PF, Bungay PM, Hsiao JK, Ball BA, Mefford IN, Dedrick RL. Quantitative microdialysis: analysis of transients and application to pharmacokinetics in brain. $\mathrm{J}$ Neurochem. 1991;57(1):103-19. 